\title{
Electronic properties of pentaorgano [60]fullerenes under an external electric field
}

\author{
Sho Furutani* and Susumu Okada
}

Graduate School of Pure and Applied Sciences, University of Tsukuba, 1-1-1 Tennodai, Tsukuba, Ibaraki 305-8571, Japan

\begin{abstract}
Electronic properties of pentaorgano [60]fullerenes under an external electric field were studied using the density functional theory combined with the effective screening medium method. The pentaorgano [60]fullerenes possess a dipole moment because of their asymmetric molecular form owing to the five functionalized groups. By injecting electrons and holes into the molecule, the magnetic states of the molecule change from $S=1 / 2$ to nonmagnetic and $S=1$ triplet states for electron and hole doping, respectively. The asymmetric molecular shape causes the unusual distribution of accumulated carriers by the electric field, depending on their mutual molecular arrangement to the electric field.
\end{abstract}

Fullerenes are known for representative nanoscale carbon allotropes with hollowcage structures ${ }^{1,2)}$ which have an infinite number of possible cage topologies, $\mathrm{C}_{2 n}(n$ rages from 10 to $\infty$ except $\left.22^{2}\right)$, because of the huge number of possible arrangements of 12 pentagonal rings and the appropriate number of hexagonal rings in accordance with Euler's theorem. ${ }^{2,3)}$ The detailed electronic structures of fullerenes strongly depend on the cage size and symmetry. ${ }^{4-11)}$ Furthermore, 12 pentagonal rings embedded into the $\mathrm{sp}^{2}$ hexagonal network cause the unique and common electronic structures in their lower unoccupied states. ${ }^{8)}$ Fullerenes usually possesses the deep lowest unoccupied (LU) state compared with the other carbon allotropes and hydrocarbon molecules, making the fullerenes electron accepters in the hybrid structures with foreign atoms and molecules. The facts indicate that the fullerenes can be constituents for a variety of electronic and energy devices.

In addition to the pristine fullerenes, the hollow-cage structure with nanometer diameter allows us to synthesize further derivatives of which electronic structure not seen in the pristine fullerene. Due to the moderate chemical reactivity arising from the large curvature, fullerenes can form the various derivatives by attaching atoms ${ }^{12-15)}$ or

${ }^{*}$ E-mail address: sfurutani@comas.frsc.tsukuba.ac.jp 
functional groups ${ }^{16-23)}$ onto their covalent network. Functionalized fullerenes are known for possessing unusual electronic structure, which is totally different from that of the pristine fullerenes, making them constituent materials for the optical, optoelectronic, magnetic, and photovoltaic devises. In our previous work, a pentaorgano [60]fullerene possesses $S=1 / 2$ magnetic moment owing to an isolated pentagonal $\pi$ electron network surrounded by the functional groups. ${ }^{20)}$ As the case of pristine [60]fullerene, the pentaorgano [60]fullerene also possesses the deep LU state so that they are applicable for the electron accepters of organic thin film devices with high efficiency. ${ }^{16,17)}$ Although the experiments have steadily advanced the devices application of the functionalized fullerenes, a little is known for the fundamental mechanism of the electron accumulation into them in the device structures. Therefore, in the present work, we aim to investigate the electronic properties of pentamethyl [60]fullerene $\left(\mathrm{C}_{60}(\mathrm{Me})_{5}\right)$ and pentaphenyl [60]fullerene $\left(\mathrm{C}_{60}(\mathrm{Ph})_{5}\right)$ under an external electric field using the density functional theory combined with the effective screening medium method. Our calculations show that $\mathrm{C}_{60}(\mathrm{Me})_{5}$ and $\mathrm{C}_{60}(\mathrm{Ph})_{5}$ possess an intrinsic dipole moment of 1.7 and $0.9 \mathrm{D}$, respectively, because of their asymmetric molecular shapes by attachment of the methyl and phenyl groups. According to the intrinsic dipole moment, molecular orientation to the external electric field sensitively affects the distribution of the accumulated carrier in $\mathrm{C}_{60}(\mathrm{Me})_{5}$ and $\mathrm{C}_{60}(\mathrm{Ph})_{5}$, implying an importance of the molecular orientation at the interfaces in organic semiconductors. Our results give theoretical insight into the microscopic mechanism of the carrier accumulation in pentaorgano [60]fullerenes.

All of the calculations were performed using the density functional theory $(\mathrm{DFT})^{24,25)}$ with the STATE package. ${ }^{26)}$ To express the exchange correlation potential among the interacting electrons, the local density approximation is applied with the Perdew-Wang functional form fitting to the Quantum Monte Carlo results on the homogeneous electron gas. ${ }^{27,28)}$ We use an ultrasoft pseudopotential to describe the interactions between the valence electrons and the ions generated by the Vanderbilt scheme. ${ }^{29)}$ The valence wave functions and charge density were expanded by a planewave basis set with cutoff energies of 25 and 225 Ry, respectively. The $\Gamma$ point sampling is adopted to perform the Brillouin zone integration. Structural optimization was performed until the remaining force acting on each atom was less than $5 \mathrm{mRy} / \AA$. To investigate the electronic properties of an individual pentaorgano [60]fullerene under the electric field, each pentaorgano [60]fullerene is well separated by its periodic im- 
(a)

\section{electrode}

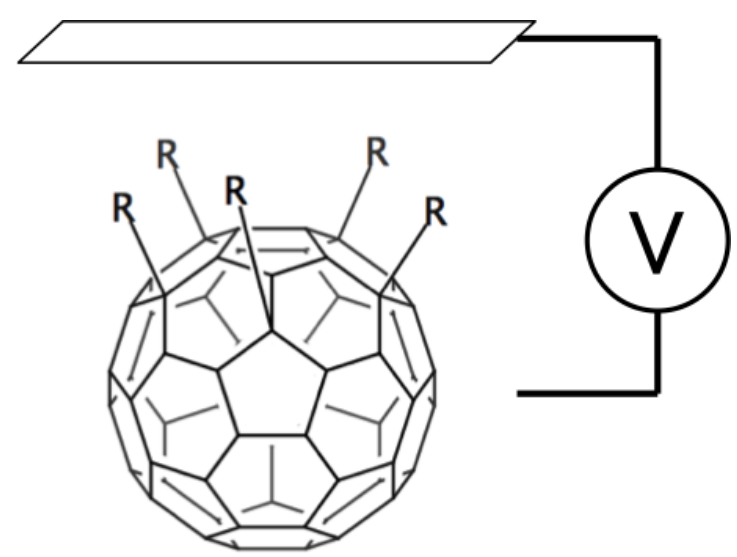

(b)

\section{electrode}

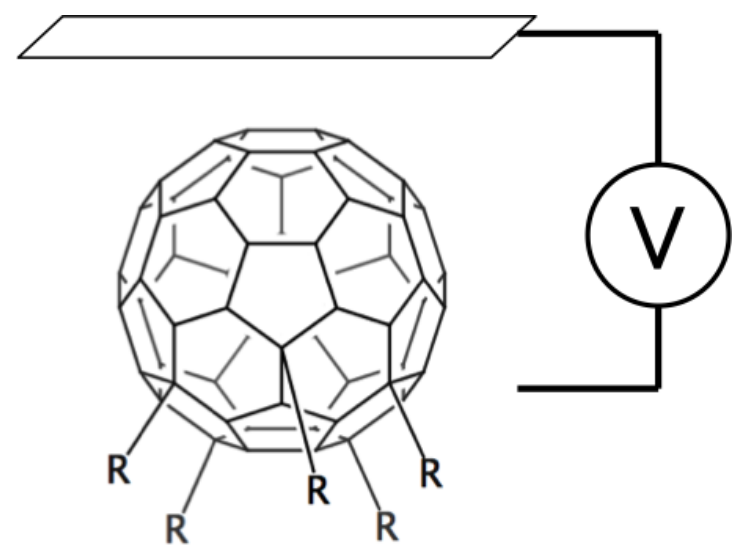

Fig. 1. A structural model of pentaorgano [60]fullerenes in field effect transistors with (a) the tail-to-head and (b) the head-to-tail molecular arrangements to the electrode.

ages under the lateral lattice parameters of $a=b=17.2$ and $23.0 \AA$ for $\mathrm{C}_{60}(\mathrm{Me})_{5}$ and $\mathrm{C}_{60}(\mathrm{Ph})_{5}$, respectively. We adopt the effective screening medium method (ESM) $)^{30}$ to solve the Poisson equation including the excess electrons or holes injected by the electrode within the framework of the DFT with the plane-wave basis set.

To simulate the pentaorgano [60]fullerenes in field effect transistors under the gate voltage, we consider a structural model shown in Fig. 1, in which the pentaorgano [60]fullerene is located below the planar gate electrode simulated by an effective screening medium to mimic an ideal metal electrode with the vacuum spacing of 4.02 and $6.18 \AA$ for $\mathrm{C}_{60}(\mathrm{Me})_{5}$ and $\mathrm{C}_{60}(\mathrm{Ph})_{5}$, respectively . To investigate the effect of the molecular arrangement on the carrier accumulation, we consider two different molecular arrangements of pentaorgano [60]fullerenes with respect to the gate electrode. For the pentaorgano [60]fullerenes, we consider pentamethyl and pentaphenyl [60]fullerenes in which five methyl or phenyl groups are attached to five atomic sites that surround pentagons situated at the apex (cap) of [60]fullerene. In accordance with the functionalization, the spherical $\pi$ electron system of [60]fullerene is divided into a bowl shape electron system consisting of $50 \pi$ electrons and a pentagonal network leading to a radical spin of $S=1 / 2$. An electric field is applied between the electrode and the pentaorgano [60]fullerenes, injecting up to 1 electrons/holes into pentaorgano [60]fullerenes. The applied gate voltage to inject the one hole/electron is approximately $3 \mathrm{~V}$ irrespec- 
(a)

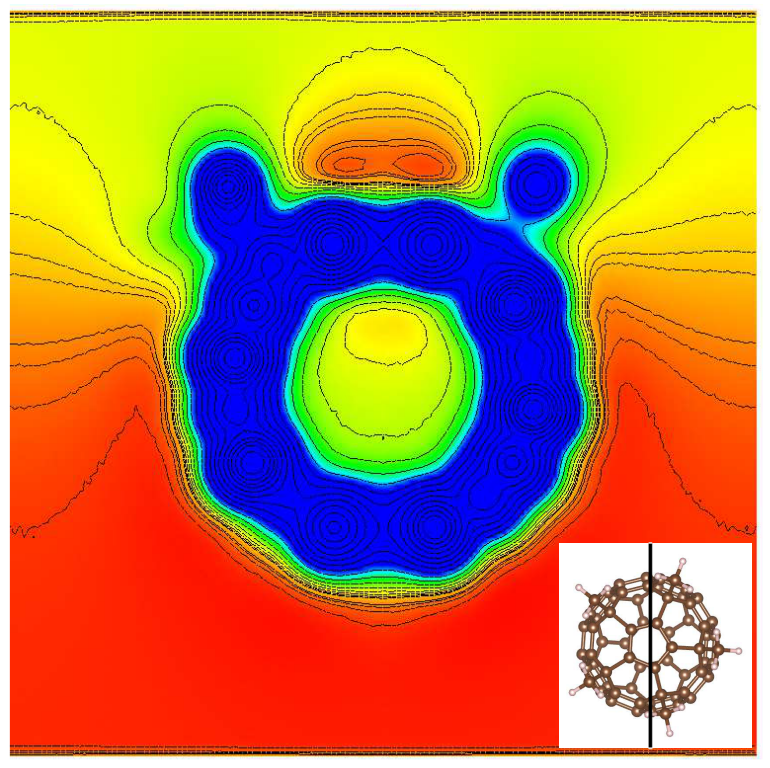

(b)

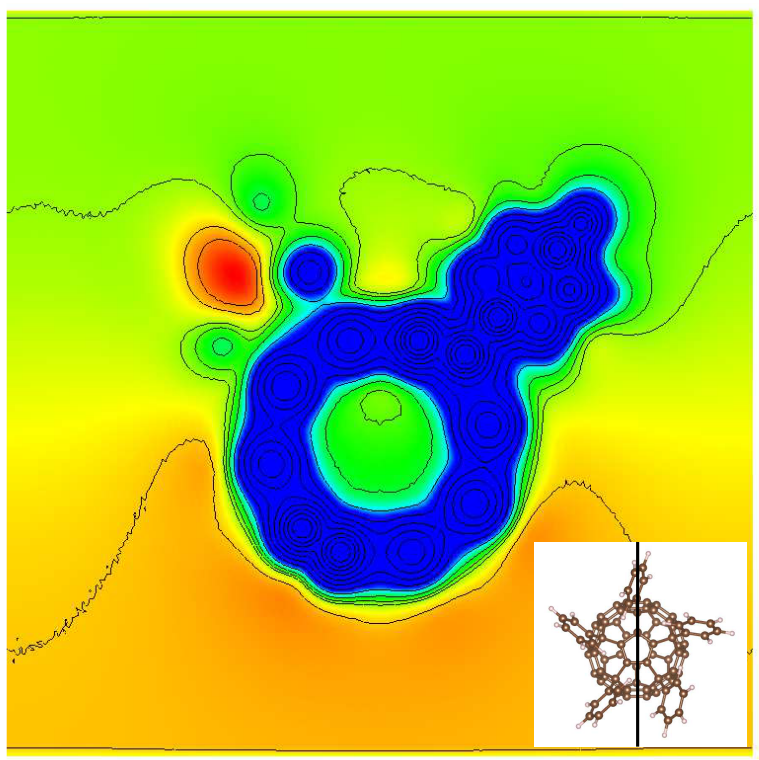

Fig. 2. Contour plots of the electrostatic potential of (a) $\mathrm{C}_{60}(\mathrm{Me})_{5}$ and (b) $\mathrm{C}_{60}(\mathrm{Ph})_{5}$ under zero electric field. In each figure, an vertical line in an inset denotes the plane where the contour plot is depicted.

tive to the functional groups and molecular arrangement. During the calculations under the conditions with a finite electric field, the geometries were kept the same as those with a zero electric field.

Figure 2 shows the electrostatic potential of $\mathrm{C}_{60}(\mathrm{Me})_{5}$ and $\mathrm{C}_{60}(\mathrm{Ph})_{5}$ under the zero electric field. The electrostatic potential at the head $\left(\mathrm{C}_{50}\right.$ bowl $)$ moiety is higher than that at the tail $\left(\mathrm{C}_{5}\right.$ ring) moiety by 0.62 and $0.35 \mathrm{~V}$ for $\mathrm{C}_{60}(\mathrm{Me})_{5}$ and $\mathrm{C}_{60}(\mathrm{Ph})_{5}$, respectively, because of the asymmetric shape of these molecules. Calculated dipole moment of $\mathrm{C}_{60}(\mathrm{Me})_{5}$ and $\mathrm{C}_{60}(\mathrm{Ph})_{5}$ are 1.7 and $0.9 \mathrm{D}$, respectively. The higher electrostatic potential is ascribed to the hydrogen atoms of methyl and phenyl groups, which are positively charged compared with the $\mathrm{C}$ atoms. Because of the $\mathrm{sp}^{2} \mathrm{C}$ atoms in the head region, the pentagonal ring causes relatively high electrostatic potential. Thus, the electrostatic properties of the pentaorgano fullerenes are sensitive to their detailed atomic structures, which may cause the orientation dependence on the carrier accumulation by the electric field.

Figure 3 shows the electronic energy level of $\mathrm{C}_{60}(\mathrm{Me})_{5}$ and $\mathrm{C}_{60}(\mathrm{Ph})_{5}$ without excess carriers. For both cases, the neutral pentaorgano fullerene has $S=1 / 2$ polarized electron spin, because of the five $\pi$ electron system at the pentagonal ring isolated by 5 functional groups. Indeed, the polarized electron spin is distributed on the pen- 
(a)

\section{up spin}

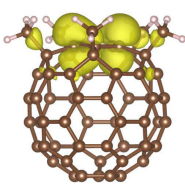

H0-1

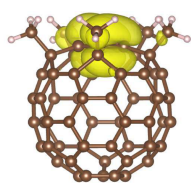

HO

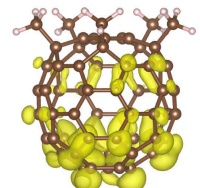

LU

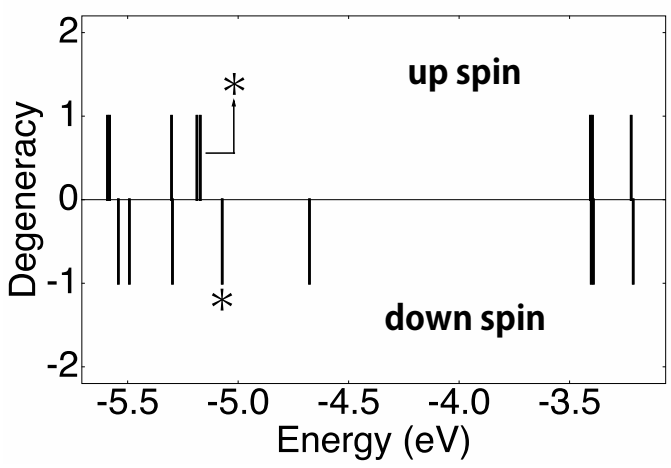

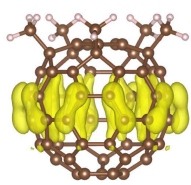

HO-1

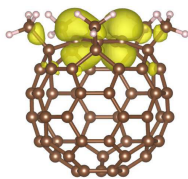

HO

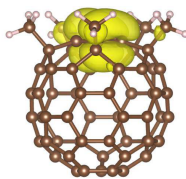

LU

down spin

(b)
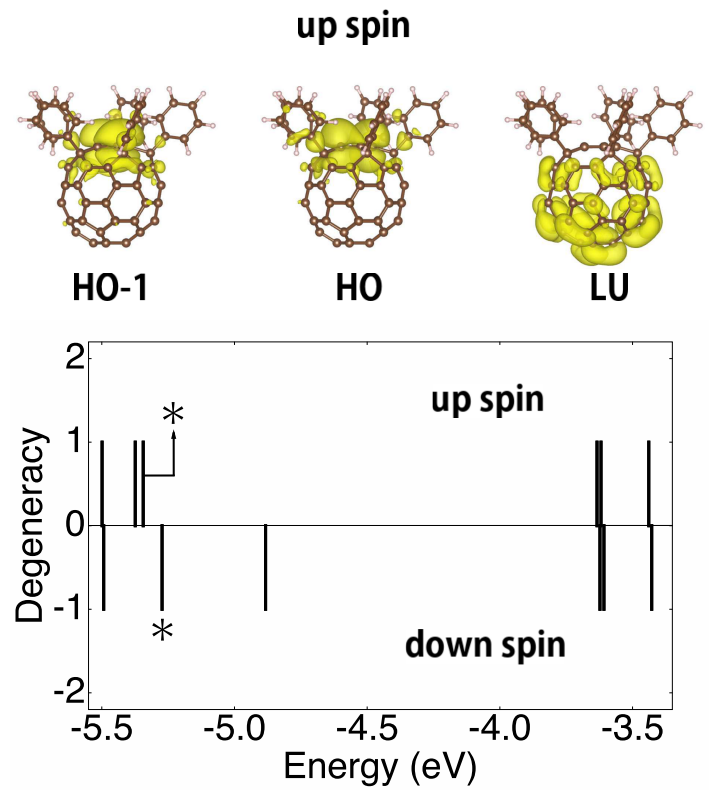

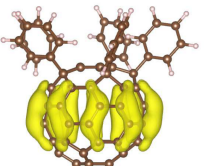

H0-1

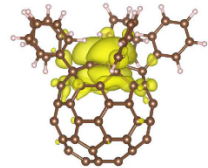

HO

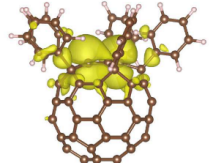

LU

down spin

Fig. 3. Electric energy levels and squared wave function near the gap between the highest occupied (HO) and the lowest unoccupied (LU) states of the of (a) $\mathrm{C}_{60}(\mathrm{Me})_{5}$ and (b) $\mathrm{C}_{60}(\mathrm{Ph})_{5}$ under zero electric field. Asterisks denote the energy level correspond to the HO state for each spin component.

tagonal ring, corresponding to the highest occupied (HO) states of the pentaorgano fullerenes. The number of polarized electron spin is sensitive to the carrier injection into the pentaorgano fullerenes: By injecting one electron into $\mathrm{C}_{60}(\mathrm{Me})_{5}$ and $\mathrm{C}_{60}(\mathrm{Ph})_{5}$, both pentaorgano [60]fullerenes did not exhibit spin polarization, because the partially occupied states associated with the pentagonal ring is fully filled by an electron. In contrast, by injecting one hole into these molecules, two electrons in the nearly two-fold degenerated states of the $\mathrm{HO}$ and $\mathrm{HO}-1$ states prefer the parallel spin arrangement, resulting in $S=1$ spin triplet state. Thus, the carrier injection can control the spin states of the pentaorgano fullerenes from $S=1 / 2$ ground state to nonmagnetic and $S=1$ states by electron and hole doping, respectively.

Figure 4 shows isosurfaces of accumulated carriers in $\mathrm{C}_{60}(\mathrm{Me})_{5}$ and $\mathrm{C}_{60}(\mathrm{Ph})_{5}$ with the head-to-tail and tail-to-head arrangement with respect to the electrode. We find 
(a)

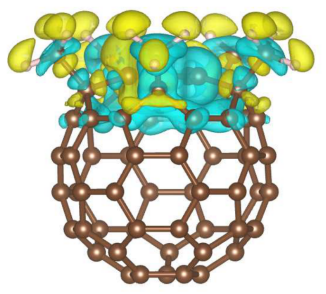

e doping

(c)

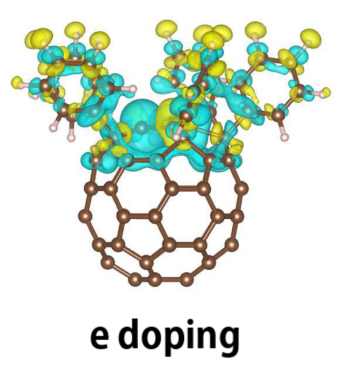

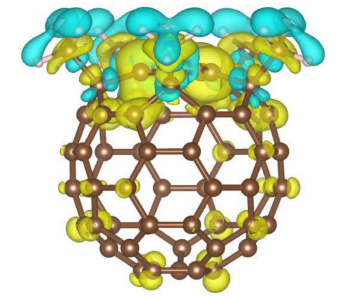

h doping (b)

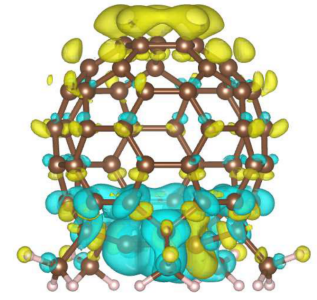

e doping

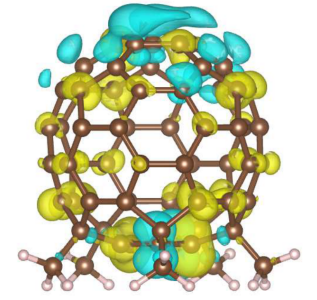

$\mathrm{h}$ doping (d)

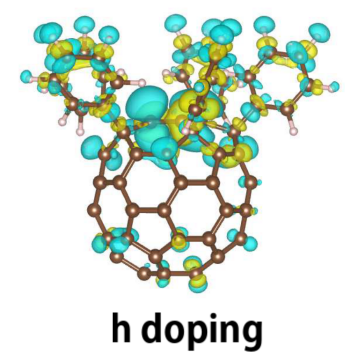

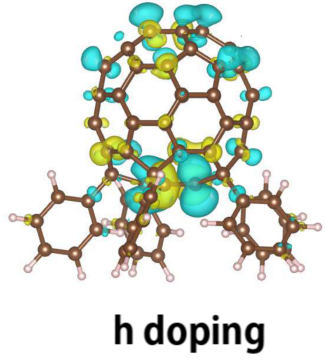

Fig. 4. Contour plots of accumulated carriers by the electric field of $\mathrm{C}_{60}(\mathrm{Me})_{5}$ with (a) tail-to-head and (b) head-to-tail molecular arrangements and those of $\mathrm{C}_{60}(\mathrm{Ph})_{5}$ with (c) tail-to-head and (d) head-to-tail molecular arrangements, repsectively, under the electron and hole concentrations of $1 e$ and $1 h$, respectively.

that the accumulated carriers are localized on the $\mathrm{C}_{5}$ ring and five attached functional groups under the tail-to-head arrangement for both electron and hole doping. In contrast, the accumulated carriers are distributed on both the top of the bowl and $\mathrm{C}_{5}$ rings under the head-to-tail arrangement, indicating that the accumulated carrier exhibits extended nature compared with the case under the tail-to-head arrangement. Furthermore, at the bowl region under the head-to-tail arrangement, the carrier density also depends on the functionalized group and injected carrier species: The carrier density at the bowl region of $\mathrm{C}_{60}(\mathrm{Me})_{5}$ is higher than that of $\mathrm{C}_{60}(\mathrm{Ph})_{5}$. The density for the electron doping is smaller than that for the hole doping. The extended nature of the accumulated carrier for the head-to-tail arrangement is ascribed to the singly occupied state at the Fermi level, which can accommodate both a hole or electron, even thought the moiety is well separated from the electrode. These facts indicate that the carrier accumulation by the electric field is sensitive to the molecular arrangements with respect to the electrode, carrier species, and functional groups. Note that the intermolecular arrangement 
(a)

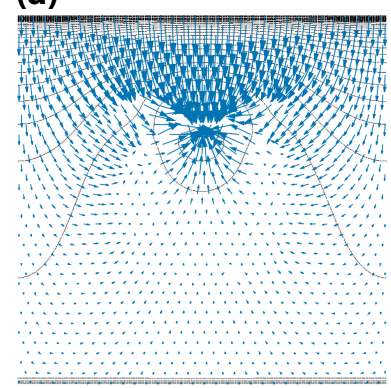

e doping

(c)

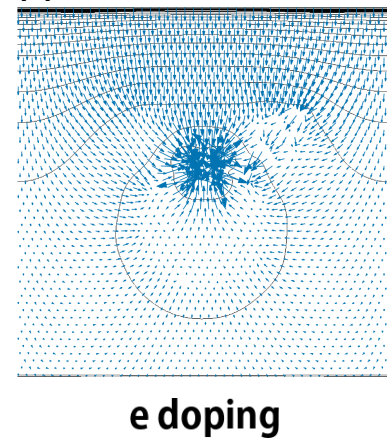

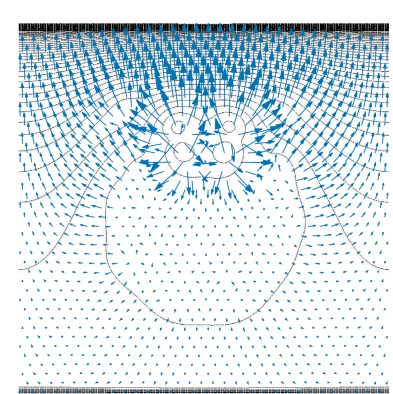

h doping

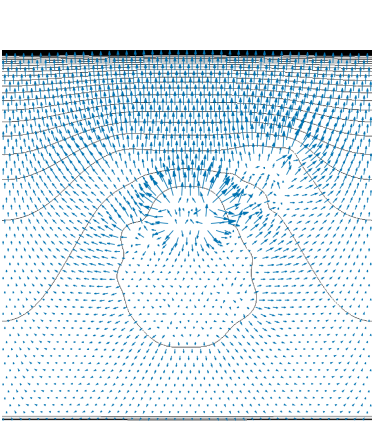

h doping (b)
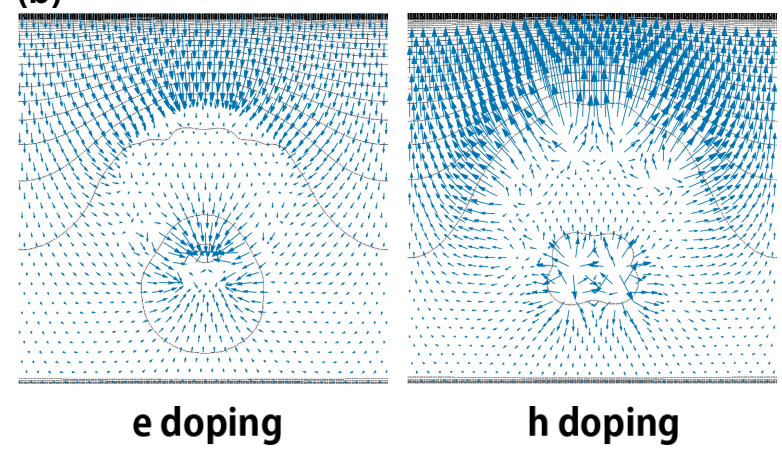

(d)

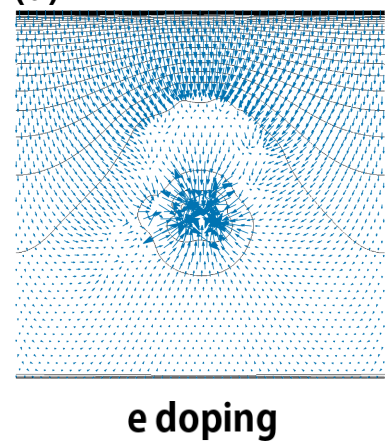

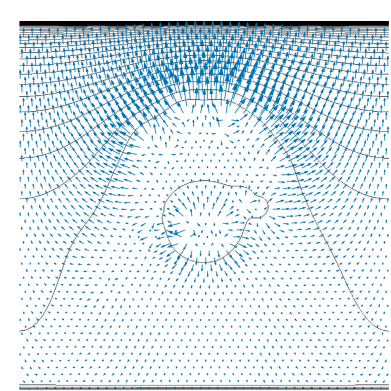

$\mathrm{h}$ doping

Fig. 5. Contour and vector plots of electrostatic potential and electric field, respectively, of $\mathrm{C}_{60}(\mathrm{Me})_{5}$ with (a) tail-to-head and (b) head-to-tail molecular arrangements and those of $\mathrm{C}_{60}(\mathrm{Ph})_{5}$ with (c) tail-to-head and (d) head-to-tail molecular arrangements, under the electron and hole concentrations of $1 e$ and $1 h$, respectively.

may cause the complicated carrier accumulation and induced electric field, because the carrier accumulation is sensitive to the molecular arrangement to the electric field.

Orientation dependence of the carrier accumulation induces the unusual electric field around the molecules under the external electric field. Figure 5 shows contour plots of electrostatic potential and vector plots of the induced electric field under the carrier injections. For the tail-to-head arrangement under both electron and hole doping, electric field concentrates around the $\mathrm{C}_{5}$ rings surrounded by five functional groups, indicating that the electric field concentrates at the interface between the pentaorgano [60]fullerenes and the organic molecules donating its electrons. In contrast, owing to the extended nature of accumulated carrier under the head-to-tail arrangement, there are two moieties where field concentration occurs: The electric field concentrates at the bowl region of the molecule where the carriers are primary accommodated. In addition to the bowl region, electric field also concentrates at the $\mathrm{C}_{5}$ ring surrounded by the methyl and phenyl groups. Accordingly, the complex electric field distribution is found 
inside the pentaorgano [60]fullerene under the head-to-tail arrangement with respect to the electrode. Thus, induced electric field around the molecule under the external electric field also depends on the molecular arrangement.

In summary, we investigated the electronic properties of $\mathrm{C}_{60}(\mathrm{Me})_{5}$ and $\mathrm{C}_{60}(\mathrm{Ph})_{5}$ under an external electric field using the density functional theory combined with the effective screening medium method. $\mathrm{C}_{60}(\mathrm{Me})_{5}$ and $\mathrm{C}_{60}(\mathrm{Ph})_{5}$ possess intrinsic dipole moment of 1.7 and $0.9 \mathrm{D}$, respectively, because of their asymmetric molecular shapes by an attachment of the methyl and phenyl groups. According to the intrinsic dipole moment as well as the distribution of the LU state which are localized at the pentagonal ring surrounded by the five functional groups, molecular orientation to the external electric field sensitively affects the distribution of the accumulated carrier in $\mathrm{C}_{60}(\mathrm{Me})_{5}$ and $\mathrm{C}_{60}(\mathrm{Ph})_{5}$, implying an importance of the molecular orientation at the interfaces in organic semiconductors. Our results give theoretical insight into the microscopic mechanism of the carrier accumulation in pentaorgano [60]fullerenes.

\section{Acknowledgement}

This work was supported by CREST, from the Japan Science and Technology Agency, JSPS KAKENHI Grant Numbers JP25246010, JP16H00898, and JP16H06331 from Japan Society For the Promotion of Science, and the Joint Research Program on ZeroEmission Energy Research, Institute of Advanced Energy, Kyoto University. Part of the calculations was performed on an NEC SX-Ace at the Cybermedia Center at Osaka University and on an SGI ICE XA/UV at the Institute of Solid State Physics, The University of Tokyo. 


\section{References}

1) M.S. Dresselhaus, G. Dresselhaus, and P.C. Eklund, Science of Fullerenes and Carbon Nanotubes (Academic Press, San Diego, CA, 1996).

2) P.W. Fowler and D.E. Manolopoulos, An Atlas of Fullerenes (Oxford University Press, Oxford, U. K., 1995).

3) D.E. Manolopoulos and P.W. Fowler, J. Chem. Phys. 96, 7603 (1992).

4) S. Saito and A. Oshiyama, Phys. Rev. Lett. 66, 2637 (1991).

5) S. Saito and A. Oshiyama, Phys. Rev. B 44, 11532(R) (1991).

6) J.R. Colt and G.E. Scuseria, J. Phys. Chem. 96, 10265 (1992).

7) K. Nakao, N. Kurita, and M. Fujita, Phys. Rev. B 49, 11415 (1994).

8) S. Saito, S. Okada, S. Sawada, and N. Hamada, Phys. Rev. Lett. 75, 685 (1995).

9) S. Okada and S. Saito, Chem. Phys. Lett. 252, 94 (1996).

10) B.L. Zhang, C.Z. Wang, and K.M. Ho, Chem. Phys. Lett, 193, 225 (1992).

11) B.L. Zhang, C.Z. Wang, K. M. Ho, C.H. Xu, and C.T. Chan, J. Chem. Phys. 98, 3095 (1993).

12) F.N. Tebbe, R.L. Harlow, D.B. Chase, D.L. Thorn, G.C. Campbell, J.C. Calabrese, N. Herron, R.J. Young, and E. Wasserman, Science 256, 822 (1992).

13) S.I. Troyanov, P.A. Troshin, O.V. Boltalina, and E. Kemnitz, Fullerenes Nanotubes Carbon Nanostruct. 11, 61 (2003).

14) J. H. Holloway, E.G. Hope, R. Taylor, G.J. Langley, A. G. Avent, T.J. Dennis, J.P. Hare, H.W. Kroto, and D.R.M. Walton, J. Chem. Soc., Chem. Commun. 12, 966 (1991).

15) R. Taylor, J.H. Holloway, E.G. Hope, A.G. Avent, G.J. Langley, T.J. Dennis, J.P. Hare, H. W. Kroto, and D. R. M. Walton: J . Chem. Soc. Chem. Commun. 9, 665 (1992).

16) Y. Matsuo and E. Nakamura, Chem. Rev. 108, 3016 (2008).

17) T, Akasaka, F. Wudl, and S. Nagase, Chemistry of Nanocarbons (Wiley, West Sussex, 2010)

18) M. Sawamura, K. Kawai, Y. Matsuo, K. Kanie, T. Kato, and E. Nakamura, Nature 419, 702 (2002).

19) Y. Matsuo, A. Muramatsu, R. Hamasaki, N. Mizushita, T. Kato, and E. Nakamura, J. Am. Chem. Soc. 126, 432 (2004).

20) S. Okada, R. Arita, Y. Matsuo, E. Nakamura, A. Oshiyama, and H. Aoki, Chem. 
Phys. Lett. 399, 157 (2004).

21) E. Nakamura, K. Tahara, Y. Matsuo, and M. Sawamura, J. Am. Chem. Soc. 125, 2834 (2003).

22) Y. Matsuo and E. Nakamura, J. Am. Chem. Soc. 127, 8457 (2005).

23) H. Nitta, Y. Matsuo, E. Nakamura, and S. Okada, Appl. Phys. Express 6, 045102 (2013).

24) P. Hohenberg and W. Kohn, Phys. Rev. 136, B864 (1964).

25) W. Kohn and L.J. Sham, Phys. Rev. 140, A1133 (1965).

26) Y. Morikawa, K. Iwata, and K. Terakura, Appl. Surf. Sci. 169-170 11 (2001).

27) J.P. Perdew and Y. Wang, Phys. Rev. B 45, 13244 (1992).

28) D.M. Ceperley and B.J. Alder, Phys. Rev. Lett. 45, 566 (1980).

29) D. Vanderbilt, Phys. Rev. B 41, 7892 (1990).

30) M. Otani and O. Sugino, Phys. Rev. B 73, 115407 (2006). 